OPEN ACCESS

Edited by:

Maria Gendron,

Yale University, United States

Reviewed by:

Yasemin Erbas,

Tillburg University, Netherlands Jefferson Becker, Pontifical Catholic University of Rio

Grande do Sul, Brazil Desirée Colombo,

University of Valencia, Spain

${ }^{*}$ Correspondence:

T. H. Stanley Seah tseah@kent.edu

Specialty section

This article was submitted to Psychology for Clinical Settings, a section of the journal

Frontiers in Psychology

Received: 29 March 2021 Accepted: 08 December 2021

Published: 10 January 2022

Citation:

Seah THS, Almahmoud S and Coifman KG (2022) Feel to Heal: Negative Emotion Differentiation Promotes Medication Adherence

in Multiple Sclerosis.

Front. Psychol. 12:687497. doi: 10.3389/fpsyg.2021.687497

\section{Feel to Heal: Negative Emotion Differentiation Promotes Medication Adherence in Multiple Sclerosis}

\author{
T. H. Stanley Seah*, Shaima Almahmoud and Karin G. Coifman \\ Department of Psychological Sciences, Kent State University, Kent, OH, United States
}

Multiple Sclerosis (MS) is a debilitating chronic autoimmune disease of the central nervous system that results in lower quality of life. Medication adherence is important for reducing relapse, disease progression, and MS-related symptoms, particularly during the early stages of MS. However, adherence may be impacted by negative emotional states. Therefore, it is important to identify protective factors. Past research suggests that the ability to discriminate between negative emotional states, also known as negative emotion differentiation (NED), may be protective against enactment of maladaptive risk-related behaviors. However, less is known as to how NED may promote adaptive health behaviors such as medication adherence. Utilizing weekly diaries, we investigated whether NED moderates the association between negative affect and medication adherence rates across 58 weeks among patients $(n=27)$ newly diagnosed with MS (following McDonald criteria). Results revealed that NED significantly moderated the relationship between negative affect and medication adherence. Specifically, greater negative affect was associated with lower adherence only for individuals reporting low NED. However, this link disappeared for those reporting moderate to high NED. Building upon past research, our findings suggest that NED may promote adaptive health behaviors and have important clinical implications for the treatment and management of chronic illness.

Keywords: emotion, emotion differentiation, health behaviors, medication adherence, multiple sclerosis, chronic illness

\section{INTRODUCTION}

Multiple Sclerosis (MS) is a chronic autoimmune disease of the central nervous system that lowers quality of life (Arnett, 2003). While there is currently no cure for MS, medication adherence is important in reducing relapse and disease progression (Goodin et al., 2002). In particular, medication adherence following initial diagnosis of MS has been found to be predictive of disease outcomes as well as future compliance rates (Kleinman et al., 2010). However, given the chronic, uncertain, and life changing nature of MS, patients often experience elevated levels of distress 
during the early stages of disease (Janssens et al., 2003). Importantly, the first 5-10 years following diagnosis are a time of considerable stress, emotional upheaval, in conjunction with frequent disruptions from changes in physical impairments and increased fatigue (Strober, 2018). Indeed, it is estimated that MS patients are at considerably high risk for psychiatric illness, with rates of lifetime depression approaching 50\% (Siegert and Abernethy, 2005). The severe consequences of depression are well-documented, contributing to expensive disruptions in functioning (Kessler et al., 2006), greater risk for physical illness (Blume et al., 2011), and within the context of chronic illness, poor treatment compliance and prognosis (Kalsekar et al., 2006; Moussavi et al., 2007). Therefore, it is important to identify protective factors that may facilitate adherence to MS and related medications.

Research suggests that emotion differentiation (ED; also known as emotional granularity), which refers to the ability to distinguish between emotional states in a fine-grained manner (e.g., fear vs. anger; Barrett et al., 2001), may be important. Individuals who are more adept at differentiating their emotions tend to label their experiences using various terms that describe the presence and intensity of specific emotions (e.g., sadness). Conversely, poorer differentiators are more likely to experience difficulties separating between emotional states and tend to describe their experiences as generally "bad" or "unpleasant" (Kashdan et al., 2015; Smidt and Suvak, 2015). In the literature, ED is commonly assessed using experience-sampling methodology, where individuals repeatedly report their emotional experiences across time. A personlevel indicator of ED is derived by calculating the intraclass correlation coefficient (ICC) between emotion ratings across the sampling duration.

Studies accumulated over the past two decades have generally shown that ED, particularly negative ED (NED), is associated with better psychosocial functioning, including lower levels of depression and anxiety (e.g., Demiralp et al., 2012; Seah et al., 2020), and decreased engagement in a wide range of maladaptive behaviors in response to negative emotion across clinical and non-clinical samples (O'Toole et al., 2020; Seah and Coifman, 2021). Indeed, there is compelling theory and evidence suggesting that NED may operate by facilitating adaptive regulation of states of distress (Kashdan et al., 2015). For example, labeling feelings precisely can decrease the intensity of emotion-related arousal (Tabibnia et al., 2008; Kircanski et al., 2012). Moreover, NED has been explicitly tied to adaptive emotion regulatory strategy use (Barrett et al., 2001; Hill and Updegraff, 2012) and has been found to mitigate the association between negative affect (NA) and maladaptive behaviors known to interfere with treatment and predict poorer prognosis, including binge drinking, social avoidance, and non-suicidal self-injury (Kashdan et al., 2010; Zaki et al., 2013; Seah et al., 2020).

While it is now increasingly clear that NED may afford some protection against maladaptive behaviors, less is known about whether it may promote adaptive health behaviors. This appears especially important in the context of chronic illness management, where negative emotional states are known to adversely impact health behaviors such as treatment adherence
(Bruce et al., 2010). Results from one prior study by Coifman et al. (2014) found that NED was positively associated with adherence in Beta-Thalassemia, a congenital blood disorder. However, this study only examined attendance at routine screenings and less is known regarding other important indicators of treatment compliance such as daily medication adherence.

Therefore, the present investigation sought to replicate and extend findings by Coifman et al. (2014) in the context of MS by examining NED as a moderator of the association between NA and self-reported medication adherence in daily life. As in previous studies, we utilized sampling methodology via weekly diaries to derive person-level indices of NED, mean NA, and medication adherence rates across a 58-week period. Given its protective benefits, we hypothesized that the effects of NA on medication adherence rates would depend on levels of NED. Specifically, we predicted that NA would be negatively associated with medication adherence at low but not high levels of NED.

\section{MATERIALS AND METHODS}

\section{Participants}

Data described in the present investigation were part of a larger study investigating emotion processing related to psychological adjustment in MS. Of the total sample, 27 participants (aged 18 and above) were included in the current study as they completed the weekly diary portion that examined emotional experiences and medication adherence in daily life over a period of 1 year. These participants were English-speaking MS patients (aged 18 and above) recruited from a local clinic and/or online advertisements from the Midwestern United States (see Eligibility Criteria and Recruitment). Participants reported a mean (SD) age of 36.11 (8.88), and were mostly female (74.1\%), White/Caucasian (81.5\%), non-Hispanic (100\%), and working full-time or part-time (51.9\%). Table 1 describes the characteristics of this sample. All participants provided informed consent prior to the start of data collection.

\section{Eligibility Criteria}

The eligibility criteria for participants in the larger study included having a diagnosis of Relapsing -Remitting MS (RRMS), largely because $85 \%$ of all MS patients are diagnosed with RRMS at disease onset (McKay et al., 2015). This diagnosis was evaluated by a neurologist according to the revised McDonald Criteria (Polman et al., 2005), which is a diagnostic scheme that provides reliable diagnoses of MS and prevents false positives. The criteria combined magnetic resonance imaging with well-established diagnostic examinations that considered neurological history and examination, and a range of other laboratory tests. Given the larger study's focus on adjustment following MS diagnosis, participants' time of MS diagnosis could not exceed 10 years prior, although nearly all $(92.6 \%)$ were diagnosed within the prior 5 years. In addition, psychiatric history was assessed in a diagnostic interview using the Structured Clinical Interview for Diagnosis of Axis I Disorders in the DSM-IV-TR (SCID-I; First et al., 2002). Participants with a history of psychosis 
TABLE 1 | Characteristics of study participants as a percentage of the sample $(n=27)$.

\begin{tabular}{lc}
\hline Characteristics & Study sample \\
\hline Sex & \\
Female & 74.1 \\
Male & 25.9 \\
Race & \\
White/Caucasian & 81.5 \\
Black/African American & 7.4 \\
Other & 11.1 \\
Ethnicity & \\
Non-Hispanic or Latino & 100 \\
Employment status & \\
Work full-time & 33.3 \\
Work part-time & 18.5 \\
Retired & 3.7 \\
Unemployed & 3.7 \\
Unemployed due to disability & 33.3 \\
Other & 7.4 \\
Date of multiple sclerosis diagnosis & \\
Within past 5 years & 14.1 \\
Within past 10 years & 3.7 \\
Diagnostic status & 7.4 \\
Met criteria for any psychological disorder & 7.4 \\
Major depressive disorder & \\
Generalized anxiety disorder & \\
Social anxiety disorder & \\
Pgoraphobia without panic disorder & \\
\hline
\end{tabular}

were excluded from the research. See Table $\mathbf{1}$ for information on individuals who met criteria for current diagnosis of any psychological disorder, as well as specific diagnoses.

\section{Recruitment}

Participants were primarily recruited from an MS clinic in Northeast Ohio. Patients who met the inclusion criteria were approached by a registered nurse who was certified by the International Organization of Multiple Sclerosis Nurses, a graduate student, or a trained research assistant. Alternatively, participants responded to advertisements posted online. Phone interviews were conducted with individuals who expressed interest in the study to evaluate eligibility and explain the study's activities. For participants who were recruited online, their diagnoses were verified (with consent) through their neurologist to ensure all eligibility criteria were still met. Sixtyone percent of participants completed the initial data collection at the MS clinic and 39\% completed it in a research lab at Kent State University. Ninety-five percent of participants were taking medications to decrease the number of relapses and reduce the progress of the disease and $5 \%{ }^{1}$ were not taking any medications for MS relapses. Fifty-five percent of participants who were taking a medication to decrease the number of

${ }^{1}$ These participants were still included in the study because they were also taking other medications for MS-related symptoms.
MS relapses were taking infused/intravenous MS medication (e.g., Tysabri and Lemtrada), 25\% were taking self-injection MS medication (e.g., Copaxone, Rebif, and Avonex), 18\% were taking oral MS medication (e.g., Tecfidera, Gilenya, and Aubagio), and $2 \%$ did not report the delivery type of their medication. In addition to the MS medication that was taken to manage the disease course, other medications (e.g., Gabapentin and Baclofen) were taken to manage MS-related symptoms. The most frequently reported symptoms were pain, such as nerve pain, muscle spasm and spasticity, and bladder problems. The time frame for participant recruitment was from June 2013 to February 2017.

\section{Procedure}

Data collection was completed across an 18-month period. However, data for this study focused exclusively on the 12month weekly sampling period. During the initial stage (first 3 months), all participants completed two lab sessions that included a diagnostic interview (described above), as well as other tasks, questionnaires, and assessments to determine physical and cognitive functioning in MS. Upon completion of the second session, participants received training to complete a weekly diary for the next 10-12 months (see "Measures" section for more details). During this stage of study participation, they received weekly diary reminders by the research team and returned each diary entry via addressed-stamped envelopes. Note that participants were provided with the option to opt out of this study component. Following completion of the diary, participants returned to the laboratory for two follow-up sessions, where they repeated assessments described in Stage 1. All study procedures were reviewed and approved (Protocol: \#13-134) by the university Institutional Review Board before the start of data collection.

\section{Measures Weekly Diary}

Participants were trained by a research assistant to complete weekly diaries to assess their emotional experiences and frequency of medication adherence. Participants completed these diaries for up to a maximum of 58 weeks (approximately 10-12month period; for 58 diaries). Participants were provided with paper copies of weekly diaries after the second laboratory session. They completed these diaries once a week, typically on the same day each week (e.g., Saturday), and returned these documents to the research team via postal mail. To increase compliance, all participants received weekly reminder phone calls and/or emails from a trained research assistant and packets of paper diaries were mailed monthly to participants. Four participants were excluded from subsequent analyses because they completed less than seven diaries in total, which was less than 1.5 standard deviations from the average number of diaries completed across the sample (Bolger et al., 2003). There were no significant demographic differences (age, sex, race/ethnicity, and employment status) between excluded individuals and the final sample. The final sample $(n=23)$ completed a mean (SD) of 37.26 (12.55) diaries (range: $12-55$; compliance: 64\%). 


\section{Momentary Self-Reported Emotions}

During each weekly diary, participants were asked to describe their current emotional experience by providing ratings on a 7-point Likert scale ranging from 1 ("none") to 7 ("strong"). Participants rated the extent to which they felt each of six negative emotion words (fear, sadness, guilt, distress, anger, disgust), which formed the NA scale. Unrelated to the present study, participants also rated how much they felt each of six positive emotion words (happiness, enjoyment, affection, surprise, amusement, relief). As in previous studies on ED, these emotion words were selected to reflect varying levels of activation across negative and positive valence dimensions of contemporary affective circumplex models (e.g., Russell, 1980; Rafaeli et al., 2007). To assess the reliability of the NA scale, we computed values at the between-person $\left(\mathrm{R}_{\mathrm{KF}}\right)$ and within-person $\left(\mathrm{R}_{\mathrm{C}}\right)$ levels following Cranford et al. (2006). The between-person $\left(R_{K F}=0.99\right)$ and within-person $\left(R_{C}=0.82\right)$ reliability for this sample were good.

\section{Mean Negative Affect}

An index of mean NA was obtained by calculating a mean score across participants' ratings of the six negative emotion words from each diary across the sampling duration. The mean (SD) level of mean NA reported in this sample was 1.53 (0.48; range: 1.01-2.79).

\section{Negative Emotion Differentiation}

As in previous studies, a person-level index of NED is obtained for each participant by computing the average ICC with absolute agreement between negative emotion ratings across all diaries (Kashdan et al., 2015). This ICC index provides a measure of how similarly (i.e., level of agreement) ratings of negative emotion words vary across time. Higher ICC values would suggest similar ratings across negative emotions at any given diary entry, while lower ICCs would suggest more differentiated responses across emotion ratings. One individual had a negative ICC $(-0.02)$ and this value was changed to 0 and included in subsequent analyses (Erbas et al., 2014). ${ }^{2}$ Following existing conventions in ED research, ICC values were reverse scored (i.e., subtracted from 1) so that higher values indicated greater NED for ease of interpretation (Seah and Coifman, 2021). The mean (SD) level of NED reported in this sample was 0.34 (0.25; range: $0.03-$ 1.00 ), which is comparable to that reported in other clinical and community samples (e.g., Zaki et al., 2013; Seah et al., 2020).

\section{Medication Adherence}

During each weekly diary, participants were asked to record information about their medications (includes those that treated MS and MS-related symptoms) over the past week and indicated whether they completed (coded as 1) or missed (coded as 0 ) a dose for each day of the week. As in Coifman et al. (2014), medication adherence rates across the sampling period were derived by calculating the proportion of the total number of

\footnotetext{
${ }^{2}$ Negative ICC values are common in studies of emotion differentiation. While ICCs should range between 0 and 1 in theory, they can take on small negative values in practice due to sampling error (Cohen et al., 2003). However, rather than excluding these data, Cohen et al. (2003) recommended assigning them a value of 0 . Notably, our results remained the same even after excluding the individual with a negative ICC score for negative emotion differentiation.
}

completed vs. missed doses across all completed diaries based on the medical records of prescribed medications. The mean (SD) rate of medication adherence reported in this sample was 0.77 (0.31; range: $0-1.00)$.

\section{Data Analysis Plan}

First, we examined bivariate correlations between primary outcome variables and potential covariates (e.g., age, total number of diaries completed) in the final sample. In addition, a series of one-way ANOVAs were conducted to examine whether there were any demographic differences in medication adherence in terms of sex, race, and employment status. Next, we tested the possible moderation of the association between mean NA and medication adherence by NED using the Hayes PROCESS macro (Model 1) in IBM SPSS (v. 23). This macro runs a series of Ordinary Least Squares regressions with the centered product term representing the interaction of mean NA by NED as a predictor of medication adherence. The estimated effects reported were unstandardized regression coefficients. Statistical significance was set at 0.05 .

\section{RESULTS}

\section{Preliminary Analyses}

Results from correlational analyses revealed a significant negative association between mean NA and the total number of completed diaries, $r=-0.42, p=0.043$. Contrary to past research, mean NA was not significantly associated with medication adherence, $r=-0.22, p=0.323$. Similarly, NED was not associated with medication adherence, $r=0.14, p=0.529$ or mean NA, $r=-0.06$, $p=0.778$. No other significant correlations were observed. Next, results from one-way ANOVAs revealed no significant differences in medication adherence due to sex, $F_{(1,21)}=0.18, p=0.679$, race, $F_{(2,20)}=0.38, p=0.687$, or employment status, $F_{(5,17)}=0.97$, $p=0.462$.

\section{Primary Analyses}

Results from the moderation analyses are presented in Table 2. As hypothesized, we found that NED significantly moderated the relationship between mean NA and medication adherence, $B=1.12, \Delta R^{2}=0.18, F_{(1,19)}=4.62, p=0.045,95 \%$ CI $[0.03$;

TABLE 2 | Significant Two-way (Mean NA by NED) interaction predicting medication adherence $(n=23)$.

\begin{tabular}{lccccccc}
\hline & Predictor & $\boldsymbol{B}$ & SE & $\mathbf{9 5 \%} \mathbf{C l}$ & $\mathbf{s r}^{\mathbf{2}}$ & $\boldsymbol{R}^{\mathbf{2}}$ & $\boldsymbol{\Delta R}^{\mathbf{2}}$ \\
\hline Step 1 & Mean NA & -0.13 & 0.14 & -0.43 to 0.16 & 0.04 & 0.06 & \\
& NED & 0.16 & 0.16 & -0.41 to 0.72 & 0.02 & & \\
Step 2 & Mean NA & $-0.63^{\star}$ & 0.27 & -1.19 to -0.08 & 0.23 & 0.25 & $0.18^{*}$ \\
& NED & -1.49 & 0.80 & -3.17 to 0.20 & 0.13 & & \\
& Mean NA $\times$ NED & $1.12^{*}$ & 0.52 & 0.03 to 2.22 & 0.18 & & \\
& $F_{(3,19)}=2.06$, & & & & & & \\
& $p=0.139$ & & & & & &
\end{tabular}

$B$, unstandardized coefficient; SE, standard error; $C l$, confidence interval; $N A$, negative affect; NED, negative emotion differentiation. ${ }^{*} p<0.05$. 
2.22], $s r^{2}=0.18$. This suggests that the impact of mean NA on medication adherence depended on levels of NED. To examine the effects of the interaction, predicted values were plotted for individuals at the mean and \pm 1 SD from the mean of NED and mean NA (refer to Figure 1). Follow-up tests of the simple slopes revealed that the association between mean NA and medication adherence under low NED (1 SD below the mean) was significantly different from zero, $b=-0.53, p=0.029$. Therefore, among individuals with low NED, mean NA predicted poorer adherence. However, the association between mean NA and medication adherence for individuals reporting moderate (at the mean; $b=-0.25, p=0.087$ ) to high ( $1 \mathrm{SD}$ above the mean; $b=0.03, p=0.859)$ NED was not significantly different from zero. Thus, mean NA did not appear to influence medication adherence rates among individuals with moderate to high NED. Finally, we re-ran our analyses controlling for the total number of completed diaries, as well as diagnostic status (i.e., individuals with vs. without a current diagnosis of a psychological disorder), and the pattern of results remained the same.

\section{DISCUSSION}

Our findings build upon past research demonstrating the protective effects of NED. Specifically, the results suggest that beyond its negative association with maladaptive behaviors, NED may also facilitate adaptive health behaviors, such as daily medication adherence. Moreover, these results held even after controlling for important covariates such as number of completed diaries and diagnostic status. Critically, our sample comprised patients who were recently diagnosed with MS, a period of elevated stress for most new patients, and suggests that NED affords protection even in such highly aversive contexts. This appears especially important given that treatment compliance reduces symptom exacerbations in MS, and in so doing, may help to improve patients' health in addition to psychological well-being and quality of life (Khayyat et al., 2019; Peacock et al., 2021). Finally, these results replicate prior findings associating NED with treatment compliance in patients with a congenital blood disorder (Coifman et al., 2014) and reinforce the importance of considering affective processes in chronic disease management.

Notably, the interaction term between NED and mean NA accounted for $18 \%$ of the variance in our model, suggesting that it may be most important to target emotion-related processes in patients showing elevated negative emotion in order to boost medication adherence in MS treatment. This is consistent with a growing body of work that has demonstrated the implicit emotion regulatory benefits of affect labeling, where assigning labels to one's emotions may facilitate downregulation of NA and psychophysiological indices of distress (e.g., amygdala activation; Torre and Lieberman, 2018). Moreover, past research suggests that ED may counteract maladaptive cognitive-emotional processes such as rumination that often exacerbate NA and increase propensity of maladaptive behavioral engagement (Zaki et al., 2013; Seah et al., 2020). Instead, it is possible that ED may enable one to disengage from difficult experiences (rather than staying "stuck"), and in turn facilitate greater psychological distance and adaptive regulation of negative emotion (Kross and Ayduk, 2017; Seah et al., 2021). Nevertheless, these hypotheses remain preliminary and future research should aim to explicitly test possible underlying mechanisms of ED in relation to adaptive behavioral response to increase our understanding of how it may operate.

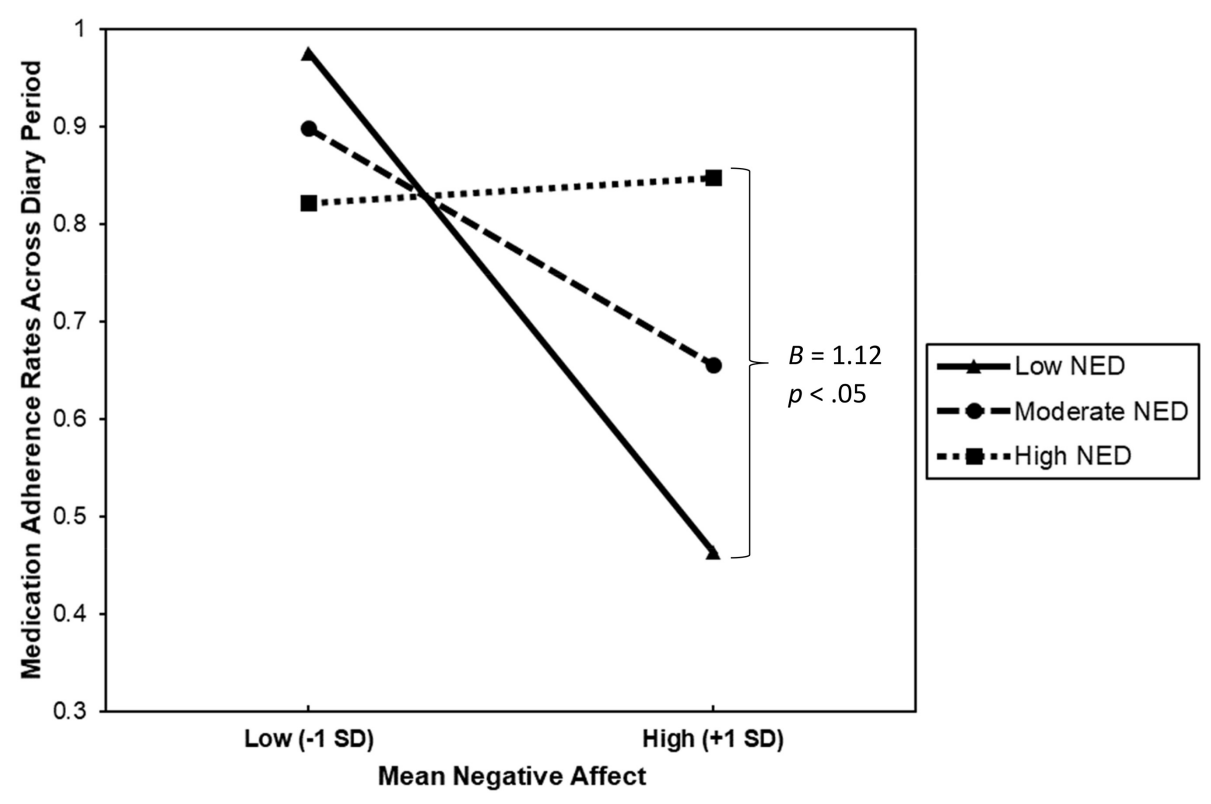

FIGURE 1 | This figure describes the interaction between mean negative affect and negative emotion differentiation (NED) when predicting medication adherence rates across the 58-week diary period among 23 patients with Multiple Sclerosis. 
The findings from the current study also have clinical implications. Our results suggest that fostering the ability to differentiate and label emotions may have potential to help patients who exhibit elevated negative emotion and distress while experiencing challenges with medication adherence. Indeed, this is a skill commonly addressed in psychotherapy (Greenberg, 2004), such as dialectical behavior therapy (Linehan, 1993), where explicit training in improving patients' understanding of emotion is associated with reductions in more severe life-threatening behaviors (e.g., suicidal behaviors; DeCou et al., 2019). Given the relatively high incidence and prevalence of common psychiatric disorders in MS (e.g., mood and anxiety disorders; McKay et al., 2018), clinicians may consider identifying patients at-risk for poor adherence and providing referrals as appropriate. While our results are largely preliminary, there is potential for patients to benefit broadly from interventions aimed at developing ED skills nonetheless.

Additionally, that NED, which is derived from more subjective patient-reported outcomes, seem to be able to impact more objective clinical behavioral outcomes such as medication adherence highlights the need to consider psychological factors in medical contexts. Indeed, the effects of psychological factors such as emotion (e.g., fear) on health behaviors (e.g., health screening) have been well-documented in the literature (see review by Consedine and Moskowitz, 2007). This appears particularly important given the chronic and uncertain nature of diseases such as MS, which inflicts significant burden on patients over time. Moreover, compliance with medication regimen is likely impacted by potential negative medication side effects (e.g., pain from injections), as well as other contextual factors (e.g., fear associated with hospital visits; Lynd et al., 2018). Our findings suggest that it may be beneficial for healthcare providers to consider patient-reported outcomes as part of patient care, which may facilitate identification of risk and/or protective factors during the course of treatment.

The results from the present investigation should be considered in light of the following strengths and limitations. First, this was a relatively rare investigation of NED in a high-risk population in relation to an essential and highly adaptive behavioral response to stress (medication adherence during chronic illness). The assessment of both NED and medication adherence was robust and took place over a yearlong period of sampling. Most research estimates NED from far shorter periods of time and has almost exclusively targeted maladaptive behavioral responses (Seah and Coifman, 2021). However, our study sample was small and had limited diversity in race/ethnicity and gender. Therefore, our results may not generalize to other racial/ethnic minority groups and nonfemale-identifying individuals. Despite the small sample, it is important to note that although not a rare disease, MS is still much less common than many other chronic illnesses and thus the total population of MS patients is also small (Evans et al., 2013). As such, our study's findings remain clinically meaningful and would benefit from future replications in larger and more diverse samples. In the present study, we assessed medication adherence broadly which limited our ability to capture variability in frequency and method of dosing for both MS and MS-related medications. Indeed, there is evidence that certain MS medications (e.g., Lemtrada; Barclay et al., 2019) have considerable variability in dosing and demand for adherence. Moreover, recall bias is possible since adherence was assessed via weekly retrospective self-report. However, it was also important to minimize participant burden particularly given the long duration of sampling ( $\sim 12$ months). Nevertheless, future research should consider more sensitive measurements of medication adherence. Finally, the correlational nature of our study prevents conclusive interpretations regarding causality. However, accumulating evidence from experimental studies on ED suggests that it plays a causal role in facilitating adaptive behavioral responses in laboratory provocations (Kircanski et al., 2012; Cameron et al., 2013). This remains an important question worthy of future exploration.

Despite its limitations, the current study revealed important findings that highlight the protective benefits of NED in the context of adjustment to chronic stressors such as MS. Specifically, our findings suggest that NED may facilitate enactment of adaptive health behaviors like medication adherence in patients with elevated distress, that may in turn improve patient health. The clinical implications of our results are also apparent, particularly given the uncertain and life changing nature of chronic illnesses like MS. Future replications in larger, more diverse clinical samples would bolster our findings with potential implications for improving the identification of psychological factors that may facilitate greater adaptation to life stress within vulnerable populations.

\section{DATA AVAILABILITY STATEMENT}

The original contributions presented in the study are publicly available. This data can be found here: https://osf.io/h5kyf/.

\section{ETHICS STATEMENT}

The studies involving human participants were reviewed and approved by the Kent State University Institutional Review Board. The patients/participants provided their written informed consent to participate in this study.

\section{AUTHOR CONTRIBUTIONS}

TS and KC analyzed and interpreted the results. TS drafted the initial manuscript. All authors contributed to study design, revisions of the manuscript, and approved the submitted version.

\section{FUNDING}

This investigation was supported (in part) by a PILOT Award from the National Multiple Sclerosis Society to KC. 


\section{REFERENCES}

Arnett, P. A. (2003). "Neuropsychological presentation and treatment of demyelinating disorders," in Handbook of Clinical Neuropsychology, eds U. K. P. Halligan and J. Marshall (Oxford: Oxford University Press), 528-543.

Barclay, K., Carruthers, R., Traboulsee, A., Bass, A. D., LaGanke, C., Bertolotto, A., et al. (2019). Best practices for long-term monitoring and follow-up of alemtuzumab-treated MS patients in real-world clinical settings. Front. Neurol. 10:253. doi: 10.3389/fneur.2019.00253

Barrett, L. F., Gross, J., Christensen, T. C., and Benvenuto, M. (2001). Knowing what you're feeling and knowing what to do about it: mapping the relation between emotion differentiation and emotion regulation. Cogn. Emot. 15, 713-724. doi: 10.1080/02699930143000239

Blume, J., Douglas, S. D., and Evans, D. L. (2011). Immune suppression and immune activation in depression. Brain Behav. Immun. 25, 221-229. doi: 10. 1016/j.bbi.2010.10.008

Bolger, N., Davis, A., and Rafaeli, E. (2003). Diary methods: capturing life as it is lived. Ann. Rev. Psychol. 54, 579-616. doi: 10.1146/annurev.psych.54.101601. 145030

Bruce, J. M., Hancock, L. M., Arnett, P., and Lynch, S. (2010). Treatment adherence in multiple sclerosis: association with emotional status, personality, and cognition. J. Behav. Med. 33, 219-227. doi: 10.1007/s10865-010-9 $247-y$

Cameron, C. D., Payne, B. K., and Doris, J. M. (2013). Morality in high definition: emotion differentiation calibrates the influence of incidental disgust on moral judgments. J. Exp. Soc. Psychol. 49, 719-725. doi: 10.1016/j.jesp.2013.02.014

Cohen, J., Cohen, P., West, S. G., and Aiken, L. S. (2003). Applied Multiple Regression/Correlation Analysis for the Behavioral Science, 3rd Edn. Mahwah, NJ: Lawrence Erlbaum Associates Publishers.

Coifman, K. G., Ross, G. S., Kleinert, D., and Giardina, P. (2014). Negative affect differentiation and adherence during treatment for thalassemia. Int. J. Behav. Med. 21, 160-168. doi: 10.1007/s12529-012-9277-7

Consedine, N. S., and Moskowitz, J. T. (2007). The role of discrete emotions in health outcomes: a critical review. App. Prevent. Psychol. 12, 59-75. doi: 10.1016/j.appsy.2007.09.001

Cranford, J. A., Shrout, P. E., Iida, M., Rafaeli, E., Yip, T., and Bolger, N. (2006). A procedure for evaluating sensitivity to within-person change: can mood measures in diary studies detect change reliably? Pers. Soc. Psychol. Bull. 32, 917-929. doi: 10.1177/0146167206287721

DeCou, C. R., Comtois, K. A., and Landes, S. J. (2019). Dialectical behavior therapy is effective for the treatment of suicidal behavior: a meta-analysis. Behav. Ther. 50, 60-72. doi: 10.1016/j.beth.2018.03.009

Demiralp, E., Thompson, R. J., Mata, J., Jaeggi, S. M., Buschkuehl, M., Barrett, L. F., et al. (2012). Feeling blue or turquoise? emotional differentiation in major depressive disorder. Psychol. Sci. 23, 1410-1416. doi: 10.1177/ 0956797612444903

Erbas, Y., Ceulemans, E., Lee, Pe, M., Koval, P., and Kuppens, P. (2014). Negative emotion differentiation: its personality and well-being correlates and a comparison of different assessment methods. Cogn. Emot. 28, 1196-1213. doi: 10.1080/02699931.2013.875890

Evans, C., Beland, S. G., Kulaga, S., Wolfson, C., Kingwell, E., Marriott, J., et al. (2013). Incidence and prevalence of multiple sclerosis in the Americas: a systematic review. Neuroepidemiology 40, 195-210. doi: 10.1159/000342779

First, M. B., Spitzer, R. L., Gibbon, M., and Williams, J. B. W. (2002). Structured Clinical Interview for DSM-IV-TR Axis I Disorders, Research Version, Patient Edition. New York: Biometrics Research, New York State Psychiatric Institute.

Goodin, D. S., Frohman, E. M., Garmany, G. P., Halper, J., Likosky, W. H., and Lublin, F. D. (2002). Disease modifying therapies in multiple sclerosis. Neurology 58, 169-178.

Greenberg, L. S. (2004). Emotion-focused therapy. Clin. Psychol. Psychother. $11,3-16$.

Hill, C. L. M., and Updegraff, J. A. (2012). Mindfulness and its relationship to emotional regulation. Emotion 12, 81-90. doi: 10.1037/a0026355

Janssens, A. C., van Doorn, P. A., de Boer, J. B., Kalkers, N. F., van der Meché, F. G., Passchier, J., et al. (2003). Anxiety and depression influence the relation between disability status and quality of life in multiple sclerosis. Mult. Scler. J. 9, 397-403. doi: 10.1191/1352458503ms930oa
Kalsekar, I. D., Madhavan, S. S., Amonkar, M. M., Makela, E. H., Scott, V. G., Douglas, S. M., et al. (2006). Depression in patients with type 2 diabetes: impact on adherence to oral hypoglycemic agents. Ann. Pharmacother. 40, 605-611. doi: 10.1345/aph.1G606

Kashdan, T. B., Barrett, L. F., and McKnight, P. E. (2015). Unpacking emotion differentiation: transforming unpleasant experience by perceiving distinctions in negativity. Curr. Dir. Psychol. Sci. 24, 10-16. doi: 10.1177/0963721414550708

Kashdan, T. B., Ferssizidis, P., Collins, R. L., and Muraven, M. (2010). Emotion differentiation as resilience against excessive alcohol use: an ecological momentary assessment in underage social drinkers. Psychol. Sci. 21, 1341-1347. doi: 10.1177/0956797610379863

Kessler, R. C., Akiskal, H. S., Ames, M., Birnbaum, H., Greenberg, P., and Wang, P. S. (2006). Prevalence and effects of mood disorders on work performance in a nationally representative sample of US workers. Am. J. Psychiatry 163, 1561-1568. doi: 10.1176/ajp.2006.163.9.1561

Khayyat, S. M., Mohamed, M. M., Khayyat, S. M. S., Alhazmi, R. S. H., Korani, M. F., Allugmani, E. B., et al. (2019). Association between medication adherence and quality of life of patients with diabetes and hypertension attending primary care clinics: a cross-sectional survey. Qual. Life Res. 28, 1053-1061. doi: 10.1007/ s11136-018-2060-8

Kircanski, K., Lieberman, M. D., and Craske, M. G. (2012). Feelings into words: contributions of language to exposure therapy. Psychol. Sci. 23, 1086-1091. doi: 10.1177/0956797612443830

Kleinman, N. L., Beren, I. A., Rajagopalan, K., and Brook, R. A. (2010). Medication adherence with disease modifying treatments for multiple sclerosis among US employees. J. Med. Econ. 13, 633-640. doi: 10.3111/13696998.2010.52 7588

Kross, E., and Ayduk, Ö (2017). "Self-distancing: theory, research, and current directions," in Advances in Experimental Social Psychology, Vol. 55, eds J. Olson and M. Zanna (Cambridge, MA: Academic Press), 81-136.

Linehan, M. M. (1993). Cognitive-Behavioral Treatment of Borderline Personality Disorder. New York: Guilford Press.

Lynd, L. D., Henrich, N. J., Hategeka, C., Marra, C. A., Mittmann, N., Evans, C., et al. (2018). Perspectives of patients with multiple sclerosis on drug treatment: a qualitative study. Int. J. MS Care 20, 269-277. doi: 10.7224/1537-2073.20 17-109

McKay, K. A., Kwan, V., Duggan, T., and Tremlett, H. (2015). Risk factors associated with the onset of relapsing-remitting and primary progressive multiple sclerosis: a systematic review. BioMed Res. Int. 2015:817238. doi: 10. $1155 / 2015 / 817238$

McKay, K. A., Tremlett, H., Fisk, J. D., Zhang, T., Patten, S. B., Kastrukoff, L., et al. (2018). Psychiatric comorbidity is associated with disability progression in multiple sclerosis. Neurology 90, e1316-e1323. doi: 10.1212/ WNL.0000000000005302

Moussavi, S., Chatterji, S., Verdes, E., Tandon, A., Patel, V., and Ustun, B. (2007). Depression, chronic diseases, and decrements in health: results from the World Health Surveys. Lancet 370, 851-858.doi: 10.1016/S0140-6736(07)61 415-9

O’Toole, M. S., Renna, M. E., Elkjær, E., Mikkelsen, M. B., and Mennin, D. S. (2020). A systematic review and meta-analysis of the association between complexity of emotion experience and behavioral adaptation. Emot. Rev. 12, 23-38. doi: 10.1002/14651858.CD013017.pub2

Peacock, E., Joyce, C., Craig, L. S., Lenane, Z., Holt, E. W., Muntner, P., et al. (2021). Low medication adherence is associated with decline in health-related quality of life: results of a longitudinal analysis among older women and men with hypertension. J. Hypertens. 39, 153-161. doi: 10.1097/HJH.0000000000002590

Polman, C. H., Wolinsky, J. S., and Reingold, S. C. (2005). Multiple sclerosis diagnostic criteria: three years later. Mult. Scler. J. 11, 5-12. doi: 10.1191/ $1352458505 \mathrm{~ms} 1135 \mathrm{oa}$

Rafaeli, E., Rogers, G. M., and Revelle, W. (2007). Affective synchrony: individual differences in mixed emotions. Pers. Soc. Psychol. Bull. 33, 915-932. doi: 10. 1177/0146167207301009

Russell, J. A. (1980). A circumplex model of affect. J. Pers. Soc. Psychol. 39, 1161-1178. doi: 10.1037/h0077714

Seah, T. H. S., and Coifman, K. G. (2021). Emotion differentiation and behavioral dysregulation in clinical and non-clinical samples: a meta-analysis. Emotion Online ahead of print doi: 10.1037/emo0000968 
Seah, T. H. S., Aurora, P., and Coifman, K. G. (2020). Emotion differentiation as a protective factor against the behavioral consequences of rumination: a conceptual replication and extension in the context of social anxiety. Behav. Ther. 51, 135-148. doi: 10.1016/j.beth.2019.05.011

Seah, T. H. S., Matt, L. M., and Coifman, K. G. (2021). Spontaneous self-distancing mediates the association between working memory capacity and emotion regulation success. Clin. Psychol. Sci. 9, 79-96. doi: 10.1177/2167702620953636

Siegert, R. J., and Abernethy, D. A. (2005). Depression in multiple sclerosis: a review. J. Neurol. Neurosurg. Psychiatry 76, 469-475.

Smidt, K. E., and Suvak, M. K. (2015). A brief, but nuanced, review of emotional granularity and emotion differentiation research. Curr. Opin. Psychol. 3, 48-51. doi: 10.1016/j.copsyc.2015.02.007

Strober, L. B. (2018). Quality of life and psychological well-being in the early stages of multiple sclerosis (MS): importance of adopting a biopsychosocial model. Disabil. Health J. 11, 555-561. doi: 10.1016/j.dhjo.2018.05.003

Tabibnia, G., Lieberman, M. D., and Craske, M. G. (2008). The lasting effect of words on feelings: words may facilitate exposure effects to threatening images. Emotion 8, 307-317. doi: 10.1037/1528-3542.8.3.307

Torre, J. B., and Lieberman, M. D. (2018). Putting feelings into words: affect labeling as implicit emotion regulation. Emot. Rev. 10, 116-124. doi: 10.1177/ 1754073917742706
Zaki, L. F., Coifman, K. G., Rafaeli, E., Berenson, K. R., and Downey, G. (2013). Emotion differentiation as a protective factor against nonsuicidal self-injury in borderline personality disorder. Behav. Ther. 44, 529-540. doi: 10.1016/j.beth. 2013.04.008

Conflict of Interest: The authors declare that the research was conducted in the absence of any commercial or financial relationships that could be construed as a potential conflict of interest.

Publisher's Note: All claims expressed in this article are solely those of the authors and do not necessarily represent those of their affiliated organizations, or those of the publisher, the editors and the reviewers. Any product that may be evaluated in this article, or claim that may be made by its manufacturer, is not guaranteed or endorsed by the publisher.

Copyright (c) 2022 Seah, Almahmoud and Coifman. This is an open-access article distributed under the terms of the Creative Commons Attribution License (CC BY). The use, distribution or reproduction in other forums is permitted, provided the original author(s) and the copyright owner(s) are credited and that the original publication in this journal is cited, in accordance with accepted academic practice. No use, distribution or reproduction is permitted which does not comply with these terms. 